\title{
Outcome analysis of anterior decompressive surgery and fusion for cervical ossification of the posterior longitudinal ligament: report of 107 cases and review of the literature
}

\author{
Junichi Mizuno, M.D., Ph.D., And Hiroshi Nakagawa, M.D., Ph.D. \\ Department of Neurological Surgery, Aichi Medical University, Aichi, Japan
}

\begin{abstract}
Object. This study was designed to determine the surgical technique and surgery-related outcome, fusion rate, and complication of anterior decompression and fusion (with various graft materials) performed in patients with ossification of the posterior longitudinal ligament (OPLL) of the cervical spine.

Methods. Between 1980 and 1998, 107 patients with radiologically proven OPLL underwent surgery via the anterior approach for direct removal of the ossified mass. Graft materials included iliac crest in 45 cases, vertebral body (VB) in 37 cases; and interbody fusion cages in 25 cases. In four patients with three-level VB grafts and one with a two-level VB graft, anterior plates were placed. Surgery-related outcome was excellent or good in $89 \%$ and fair in $11 \%$. This clinical improvement correlated well with the severity of preoperative myelopathy. Only one patient with severe myelopathy due to extensive mixed-type OPLL developed a segmental weakness of the bilateral upper extremities. The overall fusion rate was $97 \%$. Three patients with obvious spinal instability due to pseudarthrosis required reoperation. Of the graft materials used in this series, VB grafts were the most fragile.

Conclusions. The anterior approach is an effective route for decompressing the cervical cord with OPLL. Slight asymptomatic kyphotic deformity may be encountered. Of the graft materials used in our series, VB graft was considered most fragile, and thus least optimal.
\end{abstract}

\section{KEY WORDS - posterior longitudinal ligament - ossification $\quad$ anterior approach $・$ outcome • spinal fusion}

Ossification of the posterior longitudinal ligament in the cervical spine is one of the major disorders of com-

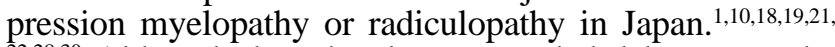
22,28,30 Although there has been extended debate over the surgical management of OPLL, direct removal of an ossified mass following corpectomy via the anterior approach is widely considered to be the optimum procedure. In OPLL, the lesion extends beyond the endplate level behind the VB, and therefore corpectomy is essential to achieve adequate decompression of the spinal cord. At the same time, reconstruction of the vertebral column is an important requirement. Graft materials including iliac crest bone, autogenous VB graft, and titanium implants

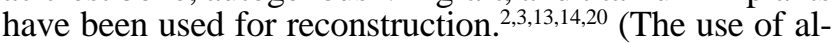
lografts is not permitted in Japan.) The microscopic technique yields good outcomes, a low risk of complications, and minimal damage to paravertebral soft tissues. ${ }^{6,7}$ In this report, we retrospectively reviewed the surgical technique used to treat patients with OPLL, placing special emphasis on surgery-related outcomes and bone fusion.

Abbreviations used in this paper: $\mathrm{ALL}=$ anterior longitudinal ligament; $\mathrm{CSF}=$ cerebrospinal fluid; $\mathrm{CT}=$ computerized tomography; $\mathrm{MR}=$ magnetic resonance; OPLL $=$ ossification of the posterior longitudinal ligament; $\mathrm{VB}=$ vertebral body.

\section{CLINICAL MATERIAL AND METHODS}

\section{Patient Population}

Between 1980 and 1998 at our institution, 146 patients underwent anterior decompression and fusion for the treatment of ossification of cervical OPLL. Of these, 39 patients with insufficient clinical or radiographic data or who attended less than 2 years of follow up were excluded from this study. There were 90 men and 17 women, who ranged in age from 36 to 74 years (mean age 62 years). In all cases the diagnosis of OPLL was histologically proven. From October 1981 to May 1992, postcorpectomy reconstruction of the spinal column was performed using iliac bone graft in 44 cases and fibula graft in one case. From June 1992 to May 1997, autogenous VBs were used as a graft material for reconstruction in 37 cases. Since June 1997, titanium interbody cages have been used in 25 cases. Forty-five patients underwent onelevel, 41 two-level, 12 three-level, four four-level, and five five-level procedures via the anterior approach.

The patients' medical records and radiological studies were reviewed, and their sex, age, clinical presentation, type of OPLL, pre- and postoperative neurological deficits, operative details, and surgery-related outcomes were recorded. Postoperative clinical outcome and presence of 


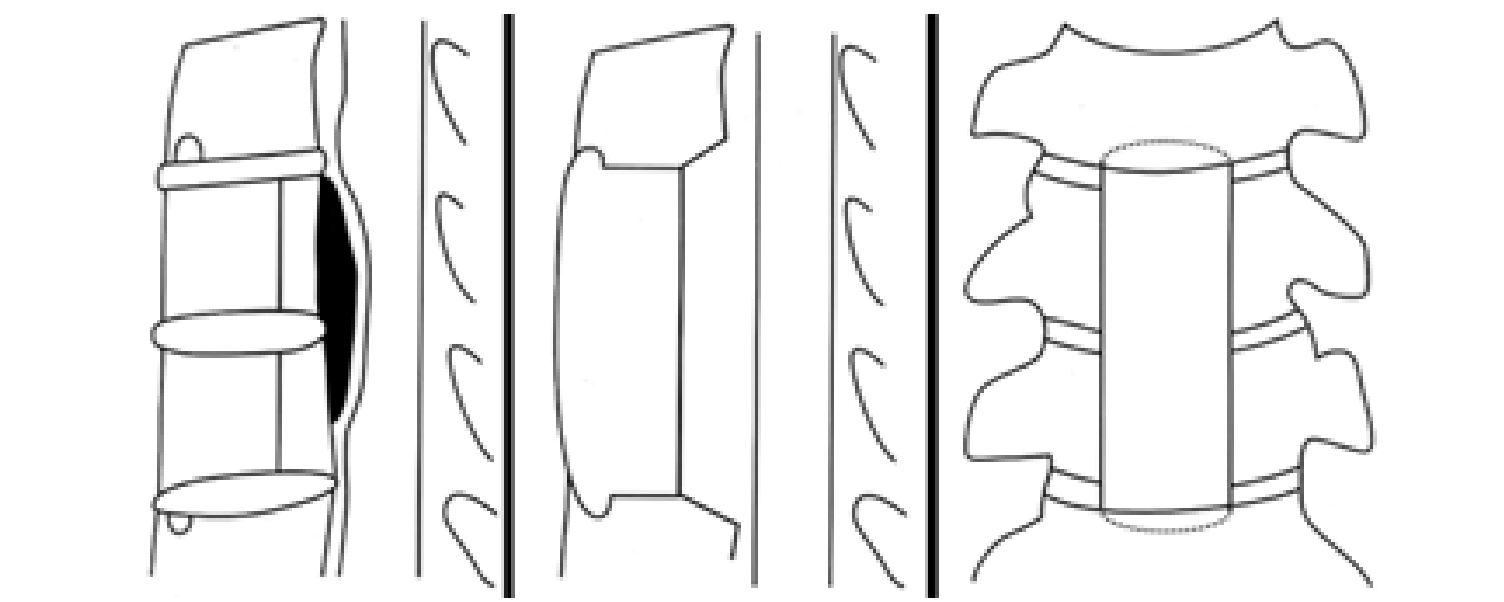

Fig. 1. Diagrams. Left: Corpectomy for removal of the OPLL. Center and Right: Insertion of iliac crest graft following excision of the OPLL.

solid fusion rate were assessed at least 6 months after the initial surgery. The interval between the onset of initial symptoms and surgery ranged from 3 days to 15 years (mean 25 months). Preoperative neurological deficits were myelopathy in 104 cases and radiculopathy in three cases.

\section{Radiological Studies}

Evaluation included plain cervical radiography, polytomography, CT scanning, myelography followed by CT myelography, and MR imaging. After MR imaging became available, myelography and CT myelography were not performed, especially in elderly patients with severe spinal canal stenosis due to OPLL. Based on evidence derived from the radiological studies, there were 90 cases of segmental OPLL, one case of continuous OPLL, and 16 cases of mixed-type OPLL.

\section{OPERATIVE TECHNIQUE}

After endotracheal intubation and induction of general anesthesia, all patients were placed in the supine position with the head slightly extended. Because the surgeons (H.N. and J.M.) are righthanded, a right-sided approach was always employed. A transverse skin-fold incision was made, beginning at the midline and crossing the anterior border of the sternocleidomastoid muscle, with a few exceptions. The appropriate surgical level was confirmed by intraoperative portable radiography.

Anterior Approach With Iliac Bone Graft. Representative diagrams and imaging studies are shown in Figs. 1 and 2. The ALL was incised, and the necessary discectomies were performed. An air drill was used to drill a trench of 15- to $18-\mathrm{mm}$ width through the anterior portion of the involved VBs. The posterior spurs were drilled out, and the OPLL was meticulously drilled to be paper thin, with the aid of a microscope. The OPLL together with the intervertebral discs and thickened posterior longitudinal ligament were then carefully separated from the underlying dura mater and were removed carefully. Anterior bulging of the dural theca and good pulsation indicated that the spinal cord was well decompressed. In cases of

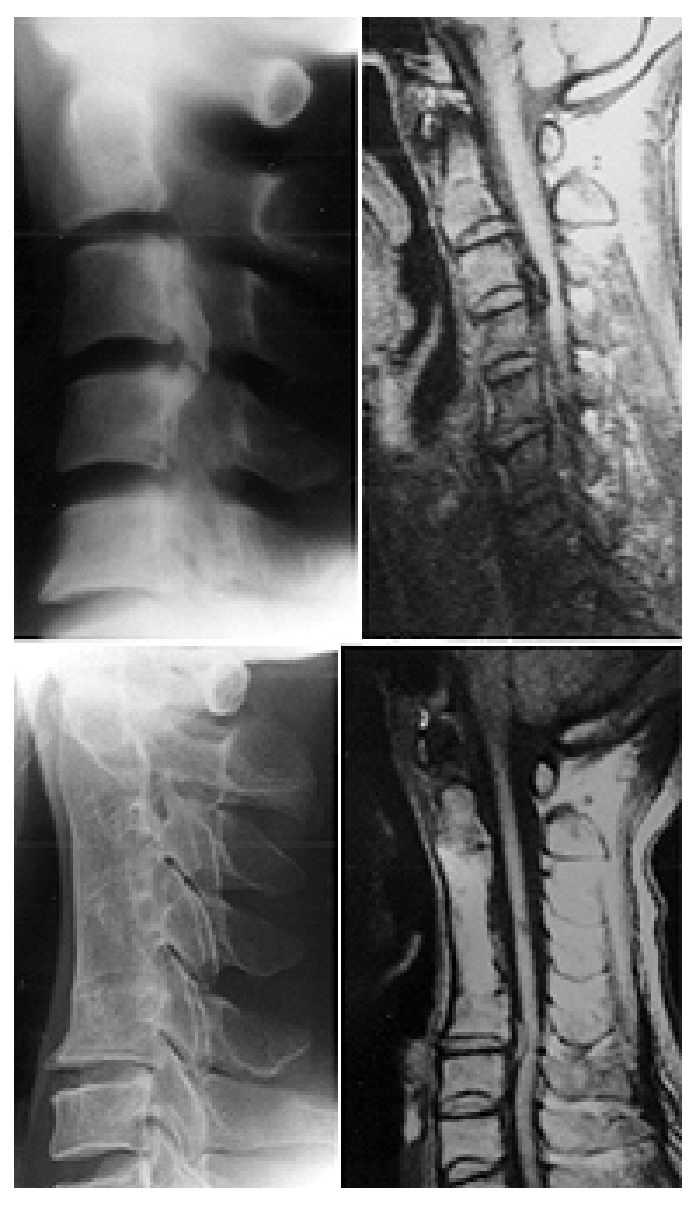

Fig. 2. Representative imaging studies. Upper Left: Lateral tomograph demonstrating mixed-type OPLL from C2-3 to C4-5. Upper Right: Sagittal $\mathrm{T}_{2}$-weighted MR image revealing severe cord compression due to OPLL. Lower Left: Postoperative radiograph demonstrating the iliac crest graft between $\mathrm{C}-2$ and $\mathrm{C}-5$ with slight anterior angulation. Lower Right: Postoperative MR image revealing a well-decompressed but atrophic cervical cord with intramedullary cavity at C3-4. 


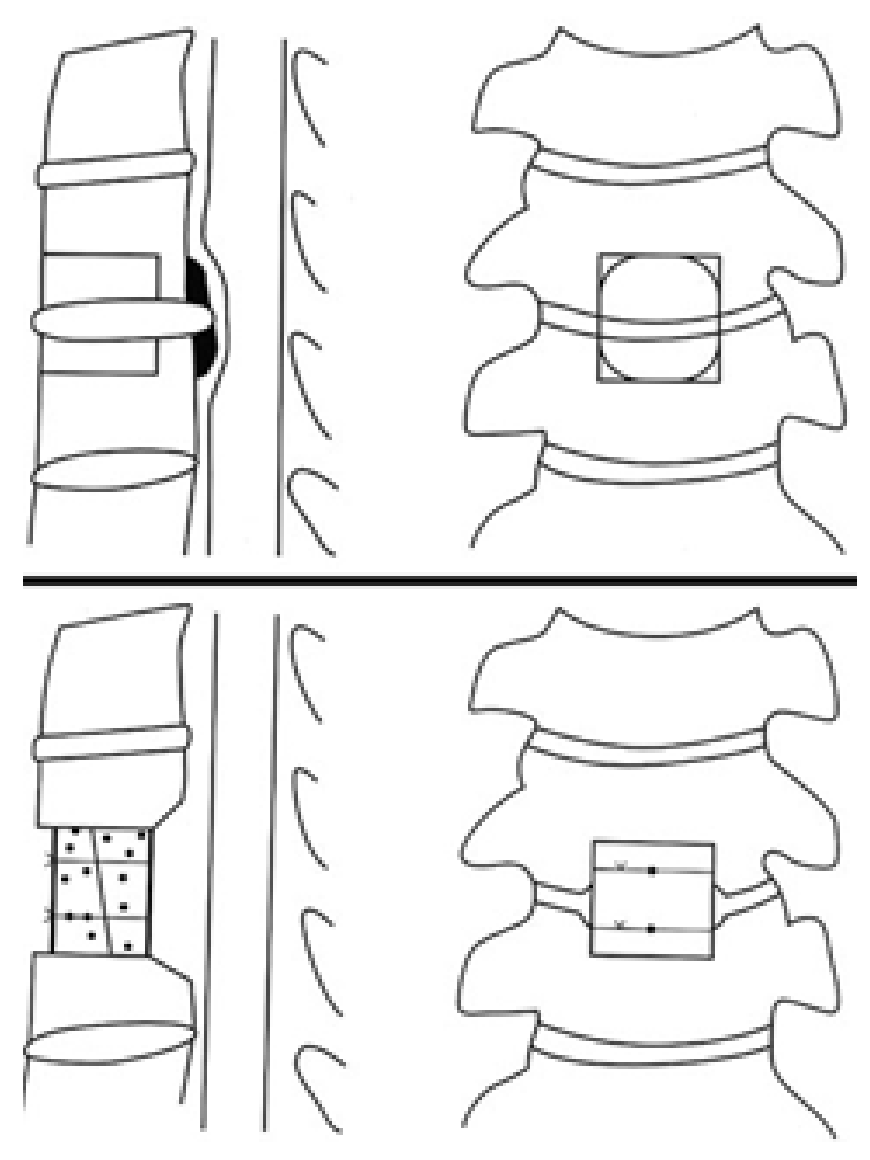

Fig. 3. Diagrams. Upper: Removal of bone graft from each VB. Lower: Insertion of VB graft following excision of the OPLL and herniated discs.

prominent OPLL, copious bleeding was carefully controlled by gentle compression with Surgicel or Oxycel. If the dura became torn or defective resulting in CSF leakage, the defect was covered with a piece of fascia, muscle, or fibrin glue. An appropriate length of full-thickness iliac bone graft was inserted as an inlay during cranial traction, and its position was confirmed after obtaining a lateral cervical radiograph.

Anterior Approach With Autogenous VB Graft. Representative diagrams and imaging are shown in Figs. 3 and 4. After incision of the ALL and the necessary discectomies, a Williams' microsurgical saw (Ace Medical Co., Los Angeles, CA) was used to perform square-shaped corpectomy. The removed vertebral bone was preserved for later use as a bone graft. The OPLL together with the residual herniated discs were separated from the dural theca and removed. Pieces of the removed vertebral bones were piled in a two-story fashion with the bone marrow portions facing each other, and sutured with nylon. The layered bone was inserted into the space between the adjacent VBs as a graft.

Small fragments of the residual bone chips were placed surrounding the graft. Fibrin glue was applied over the anterior surface of the bone graft. An additional anterior plate was used in five cases in which a two- or three-level fusion had been performed.

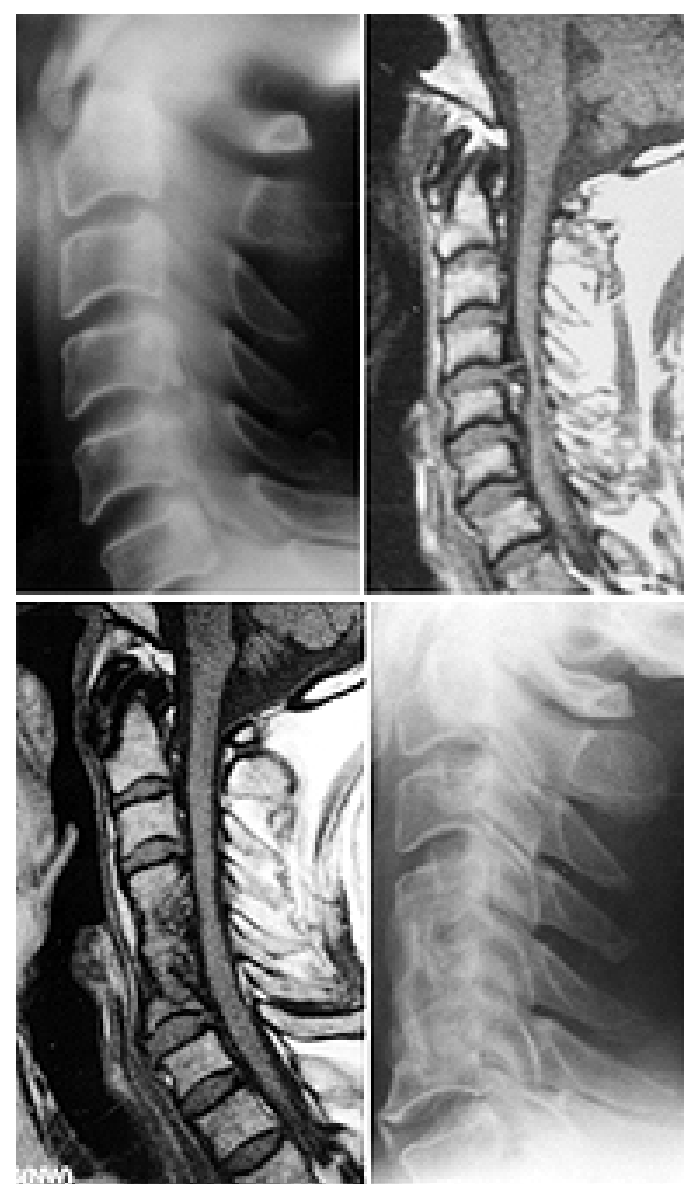

Fig. 4. Representative imaging studies. Upper Left: Lateral tomograph demonstrating mixed-type OPLL from C-3 to C-6. Upper Right: $\mathrm{T}_{1}$-weighted MR image revealing severe cord compression due to OPLL, as well as herniated disc from C-4 to C-6. Lower Left: Postoperative radiograph demonstrating a solid fusion after application of VB graft from C-4 to C-6. Lower Right: Postoperative MR image demonstrating reasonable decompression of the cord after removal of the OPLL and herniated disc material.

Anterior Approach With Interbody Cage Fixation. Representative diagrams and imaging studies are shown in Figs. 5 and 6. After the necessary discectomies, the Williams' surgical saw was used to undertake the relatively smaller corpectomy. The VBs were cut in a squareshaped fashion and were enlarged using an appropriate reamer. The OPLL and residual VBs, together with herniated discs and posterior spur, were removed through the space. The OPLL behind the mid-VBs was approached superiorly and inferiorly for total removal by changing the angle of the microscope and operating table. After confirming that good decompression (bulging and pulsatile dural theca) and complete hemostasis were achieved, tapping was performed. The interbody cage (BAK/C; Sulzer Spine Tech Minneapolis, MN, and CCM; A-Spine, Tokyo, Japan) with a 10-,12-, or 14-mm inner diameter packed with bone chips, which were derived from the corpectomy, was then placed. If bone chips were not sufficient, ceramic granules were then placed in the space of corpectomy as a graft. The segmental OPLL usually originated at the endplate level but often extended to the mid-VB, and 


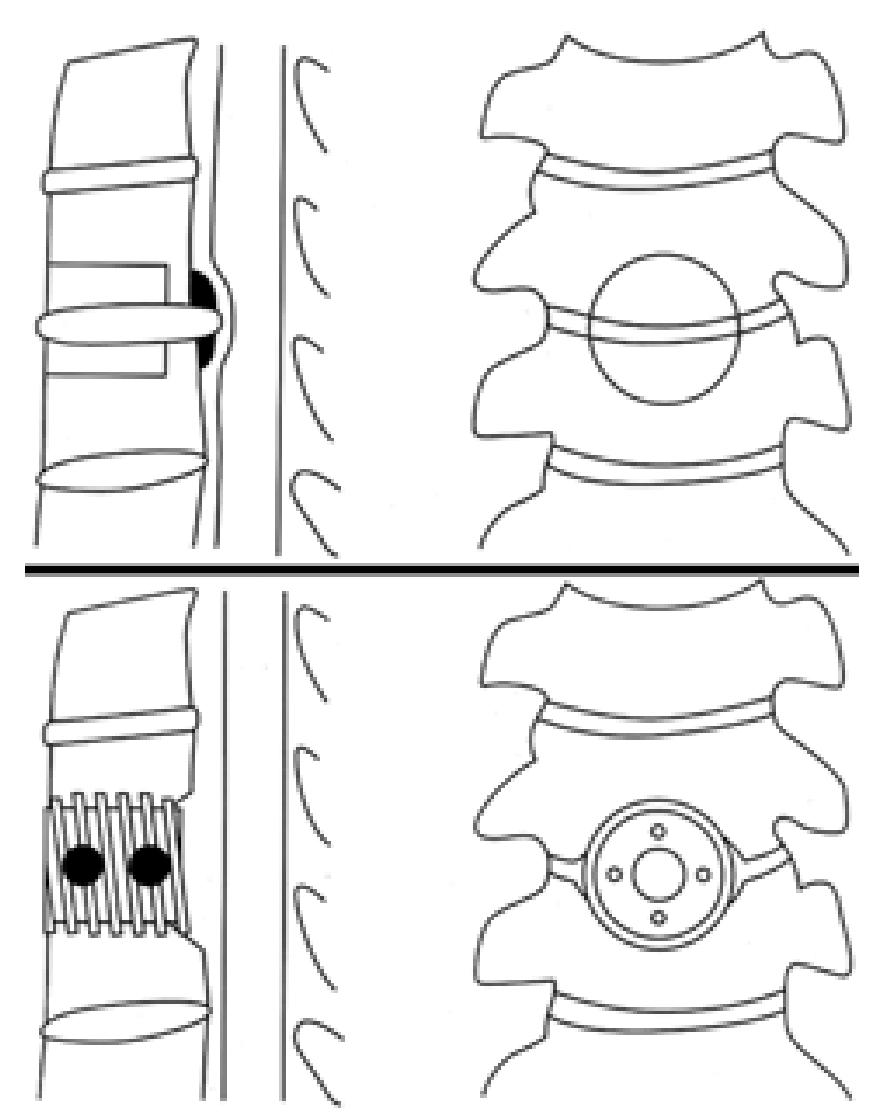

Fig. 5. Diagrams. Upper: Corpectomy and reaming of VBs. Lower: Insertion of interbody cage following excision of the OPLL and herniated disc material.

therefore the size of the cage was adjusted to the extent of corpectomy for the ossified mass. Intraoperative radiography was used to confirm the appropriate placement of the cages. Except in difficult cases, intraoperative fluoroscopy was not routinely used.

\section{Follow-Up Examination}

Immediately after surgery, routine anteroposterior and lateral plain radiographs and CT scans were obtained to assess the extent of decompression and the placement of grafts, plates, and cages. After MR imaging became available, this imaging modality was used in most cases postoperatively. All patients in this series were followed clinically and radiographically, undergoing plain and dynamic cervical spine radiography at 1,3 , and 6 months postoperatively.

\section{RESULTS}

\section{Operative Data}

Forty-five patients who underwent decompression and fusion with iliac crest graft wore a halo brace for 3 months postoperatively. No additional spinal instrumentation was required in these cases.

Thirty-seven patients who underwent decompression and fusion with a VB graft wore a cervicothoracic brace for 2 months. In three patients who underwent three-level

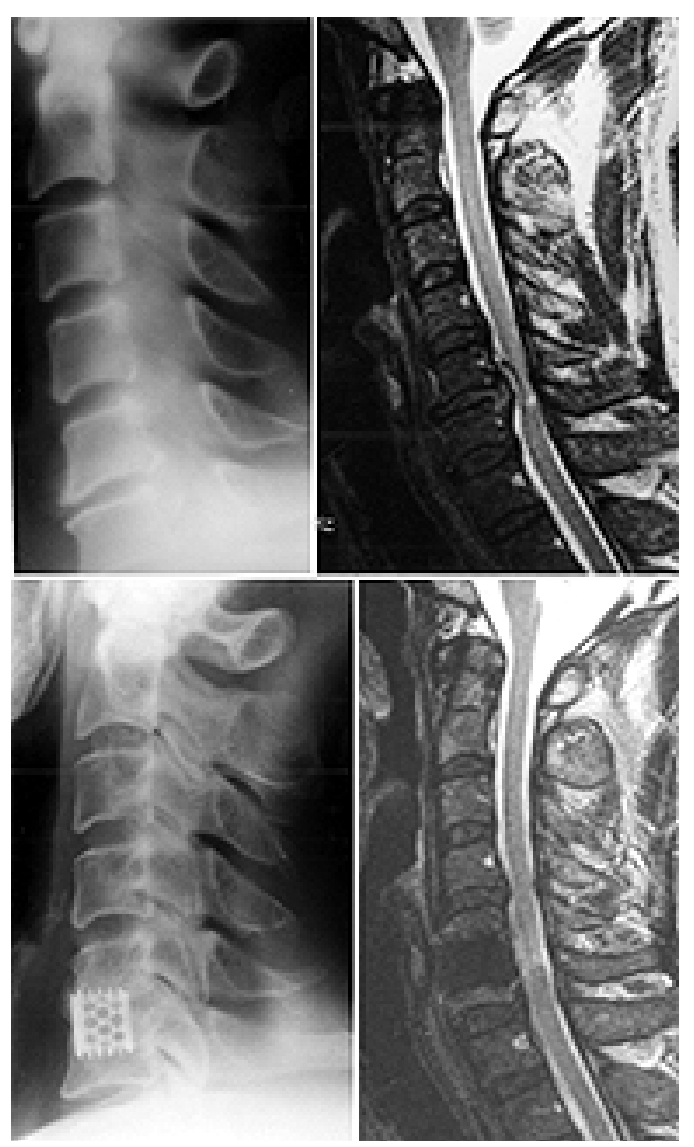

Fig. 6. Representative imaging studies. Upper Left: Lateral tomograph revealing segmental OPLL at C-5. Upper Right: Sagittal $\mathrm{T}_{2}$-weighted MR image demonstrating cord compression due to the OPLL and herniated disc; note the intramedullary high signal intensity at C5-6. Lower Left: Postoperative radiograph revealing good fusion after application of a 12-mm interbody cage at C5-6. Lower Right: Postoperative MR image demonstrating good decompression after removal of the OPLL and herniated disc material.

fusion and one patient who underwent two-level fusion, Orion plates (Medtronic Sofamor Danek, Memphis, TN) were placed to achieve further internal stabilization.

Twenty-five patients who underwent decompression and fusion in which interbody cages were used wore a soft collar for 4 to 8 weeks. No further instrumentation was required. An additional seven microdiscectomies and three transuncal foraminotomies were performed at adjacent disc levels to evacuate the midline or paramedian herniated discs or foraminal osteophytes. Bone chips, which were subsequently packed inside a cage, were collected from the corpectomy site of the VB; thus the iliac crest remained intact.

\section{Patient Outcome}

Ninety-three $(89 \%)$ of 104 patients who presented with myelopathy improved, and all three patients who presented with radiculopathy improved. Those in whom satisfactory postoperative neurological improvement was not shown had developed severe myelopathy or extensive OPLL with severe cord compression preoperatively. 
There were no significant differences in the postoperative neurological improvement when the following factors were analyzed: age, sex, surgical technique, or the number of fusion levels. Two patients experienced transient segmental neurological deterioration, and one patient, in whom extensive mixed-type OPLL with severe myelopathy was present, developed permanent weakness of the bilateral deltoid and biceps, despite sufficient improvement in long tract signs. Three patients died of the systemic disease unrelated to the OPLL.

\section{Fusion Rate}

Overall fusion was achieved in 97\% (104 of 107 patients). Pseudarthrosis-related spinal instability was obvious in three patients (one case in which iliac crest graft was used and two cases in which autogenous VB grafts were used). Cage subsidence was radiologically demonstrated in three asymptomatic patients, but no further operation was required.

\section{Intraoperative and Postoperative Complications}

Cerebrospinal fluid leakage was the most common intraoperative complication, occurring in 21 cases. The repair of the dural laceration or defect was undertaken using a muscle piece, Oxycel, and fibrin glue. A lumbar drain was installed postoperatively for 1 week in more than one third of the cases. Of these 21 cases, nerve root injuries occurred in three patients (permanent in one and transient in two); the nerve roots were exposed through the defective dura and were probably damaged when the residual small fragments of OPLL at the lateral recess were being removed and copious interligamentous sinus bleeding was being controlled. All were C-5 nerve roots in cases of large OPLL with prominent lateral extension.

There were no intraoperative vertebral artery or esophageal injuries. Although the larynx and trachea were not perforated in a single case, transient dyspnea was observed in seven cases, mostly caused by long-term retraction of the larynx or accumulation of CSF, although one case was caused by postoperative formation of a hematoma. This latter patient was treated with immediate tracheostomy, recovered fully, and returned to work. There were 12 cases with transient ipsilateral recurrent nerve palsy, and one case in which poor recovery was probably due to the pressure of ossification of ALL.

Donor-site pain (in patients in whom iliac crest grafts were used) was the most common postoperative complication. Some elderly patients experienced a prolonged rehabilitation period or independent walking difficulty because of this pain; however, after we began to use autogenous VB grafts or interbody fusion cages, this common complication was eliminated. Infection of head pins from the halo vest occurred in four cases.

There were no serious postoperative systemic medical problems such as deep venous thrombosis, pneumonia, or cardiac failure.

The most common bone graft-related complication was anterior kyphosis, occurring more commonly in patients who underwent surgery in which autogenous VB grafts were used. Cage dislodgment was not observed in any patients, although asymptomatic subsidence of adjacent cages was demonstrated on postoperative plain lateral cer- vical radiographs obtained in five patients. This phenomenon occurred in cases in which consecutive large cages had been placed at adjacent levels. Although subsidence occurred, fusion was complete, and no reoperation was necessary. Slight dislodgment of the cage was observed in two cases: one long-term hemodialysis patient and one elderly woman. A hard collar was recommended to these patients for an additional 3 months.

\section{DISCUSSION}

There are two surgical procedures for the treatment of OPLL: 1) direct removal of the ossified mass via an anterior approach or 2) decompression in which the techniques of laminectomy or laminoplasty are used, via a posterior approach. ${ }^{1,12,22}$ Although the decision should be based on patient age, severity of symptom, type of OPLL, and the surgeon's preference, we believe that this microsurgical technique can be used to achieve safe and satisfactory decompression by removing OPLL via the anterior approach. In the anterior approach, because corpectomy is necessary, fusion always becomes an important issue; however, fusion rates in series with OPLL treated via the anterior approach have been reported in only a few studies because of the rarity of this disease..$^{8,15}$

In 1958 Smith and Robinson ${ }^{27}$ described an anterior cervical approach for decompression and stabilization of segmental disease in which they placed tricortical horseshoe-shaped iliac crest grafts into the intervertebral space. In 1958 Cloward $^{4}$ used a dowel bone graft to achieve arthrodesis after performing anterior cervical decompression. Although several technical modifications have been made over the years, no clearly superior method of performing the operation has emerged. Although these classical techniques offer generally satisfying outcomes, kyphotic deformity, graft collapse, recurrent symptoms, and persistent donor-site pain may occur. To overcome these disadvantages, a comparison of graft materials, supplemental anterior plates, and external immobilization was analyzed in patients with cervical discogenic disease. ${ }^{9}$

\section{Surgical Technique}

The anterior approach with direct removal of OPLL is the procedure of choice in segmental OPLL. ${ }^{1,14,23}$ Patients with extensive continuous or mixed-type OPLL affecting more than four levels are treated via a posterior approach and laminoplasty. To determine the correct approach, it is essential to review preoperative radiological studies including plain radiography, polytomography, CT, and MR imaging. ${ }^{13,23}$ Complete corpectomy with removal of OPLL is the standard or classical anterior procedure, but this requires an iliac or fibula graft. (In Japan, allografts are not legally permitted for use in humans.) Persistent donor-site pain or discomfort annoys patients and sometimes makes walking difficult. In addition to this complication, the application of a halo brace will cause considerable discomfort and may prolong the rehabilitation period. Therefore, to resolve these problems, we began using cervical VBs as a graft material in $1992 .{ }^{13}$ Williams $^{31}$ originally described this technique in 1992, and Isu, et al., ${ }^{13}$ reported a similar technique in 1994. We modified their techniques and applied it for use in patients with OPLL. 
Although a relatively wide operating field can be obtained in this procedure-for removal of segmental OPLL as well as the associated intervertebral herniated discs and posterior osteophytes-residual OPLL located behind the mid-VB has to be meticulously resected from above and below by using undercutting, drilling, curettes, and Kerrison rongeurs while changing the angle of the microscope. This is the most difficult procedure involved in the whole technique, but it avoids donor-site complications and allows early mobilization of the patients wearing a cervicothoracic brace. One technical problem is that the cervical VB bone is sometimes of an inappropriate size or thickness, especially in heavy smokers or osteoporotic patients, and this results in anterior angulation of the cervical spine. In fact, we found postoperative anterior angulation more frequently in patients in whom VB grafts were implanted than those in whom iliac grafts were used. Isu, et al., ${ }^{13}$ reported that $4.4 \%$ of 90 patients developed postoperative anterior angulation. To prevent anterior angulation, we used additional anterior plate fixation in cases with more than three-level fusion or in cases with twolevel fusion in which the size of the graft was inappropriate. Additionally, in some cases in which the bone graft was insufficiently thick, we combined the VB and Apaceram into a sandwich-type graft. More recently we started to use titanium threaded interbody cages instead of autogenous bone grafts in patients with OPLL. This procedure provides solid stabilization, and only one patient developed slight anterior angulation postoperatively. In cases in which more than a two-level fusion is required, consecutive application of cages larger than $10 \mathrm{~mm}$ should be performed with great care to prevent subsidence.

\section{Fusion Rate}

Vertebral column instability associated with pseudarthrosis after surgery performed via an anterior approach is of major concern. The rate of pseudarthrosis ranges from 4 to $6 \%$ for one level fusions, and the rate increases in some series up to $17 \%$ when three levels are fused. ${ }^{2,3,6,7,17}$ In contrast, in 1993 Shinomiya, et al., ${ }^{26}$ studied 129 patients with OPLL and found that $16(12 \%)$ required a second operation. In 1998, Epstein ${ }^{8}$ reported that in three (47\%) of her 76 patients with cervical OPLL significant instability requiring reoperation was shown. We found three cases of postoperative kyphotic deformity $(6.7 \%)$ due to pseudarthrosis in patients receiving iliac crest grafts, and in nine cases (24\%) in patients receiving VB grafts. This difference may be related to the strength of graft material and the method of external orthosis. There were no kyphotic deformities in cases in which VB grafts were supplemented with cervical anterior plating in a three-level procedure. We conclude that autogenous VB grafting should be followed by supplemental anterior plating to prevent pseudarthrosis in cases in which the grafts are fragile.

\section{Spinal Instrumentation}

Advancement in spinal hardware and ceramics has led to increased surgical options in terms of postcorpectomy graft materials. ${ }^{9,15,29}$ The application of anterior plate fixation combined with placement of interbody fusion cages has also shown increasing popularity., ${ }^{9,25}$ The interbody fu- sion cage, shaped as a hollow cylinder, was originally designed for lumbar fusion. ${ }^{24}$ We began to use interbody cages for fusion after direct resection of the OPLL in 1997, and in only one case (4\%) has slight postoperative instability been observed. The benefits associated with a cage over the grafting techniques include: 1 ) avoidance of donor-site pain; 2) avoidance of cage collapse; and 3) early rehabilitation and return to work because of the greater internal strength supporting the cervical spinal column. ${ }^{11}$

\section{Surgery-Related Outcomes and Complications}

To our knowledge, this series of 107 patients with microsurgical corpectomy-treated OPLL is the largest to be reported in the literature. The surgery-related outcomes were reasonable and satisfactory. At the latest followup examination, only two patients needed reoperation. Growth of OPLL at a nonoperated site was demonstrated, and the patients underwent anterior decompression at the different site. Interbody fusion cages were used. Kojima, et al. ${ }^{14}$ reported that $45(87 \%)$ of their 52 patients with multiple spondylosis and OPLL improved neurologically after anterior decompression and fusion. In the series reported by Eleraky, et al., ${ }^{5} 86.5 \%$ of their patients improved neurologically after corpectomy.

The most common intraoperative complication was CSF leakage. Twenty-one patients (20\%) developed CSF leaks. This complication rate is much higher than that reported for discogenic diseases in the literature. ${ }^{5}$ This is because OPLL tends to be adherent to the underlying dura mater more frequently than other discogenic lesions. ${ }^{7,8}$ Isolated ossification of the dura mater has been reported in the literature ${ }^{16}$ and also was observed in several cases in our series. Therefore, surgeons who perform anterior-approach surgery in patients with OPLL must be careful to avoid neural damage because of possible dural defect. Only one patient in our series exhibited postoperative bilateral C-5 nerve palsy, despite improvement of the long tract sign. We speculate that the neurological deterioration in this particular case was caused by C-5 nerve damage due to manipulation of residual OPLL at the lateral recess. Other major complications such as spinal cord injury, vertebral artery injury, or visceral injury did not occur in our series.

Postoperative donor-site pain was common in patients in whom iliac crest grafts were used. This complication has repeatedly been documented in the literature. After we started to use VB grafts and fusion with an interbody fusion cages, this complication was eliminated.

\section{CONCLUSIONS}

Microsurgical anterior decompression and fusion play an important role in the optimum management of patients with OPLL. Although the procedure is technically demanding and challenging, but most complications can be avoided or resolved if the surgeon has knowledge of the nature of OPLL and uses meticulous microsurgical techniques.

Autogenous VB grafts and interbody fusion cages make collection of bone from the iliac crest unnecessary, and in using either, one can avoid causing postoperative donorsite pain and the need for a halo brace. Bone fusion was 
achieved with all graft materials used in our series, with the occurrence of slight asymptomatic kyphotic deformity. Among graft materials, VB graft was considered to be most fragile.

\section{References}

1. Abe H, Tsuru M, Ito T, et al: Anterior decompression for ossification of the posterior longitudinal ligament of the cervical spine. J Neurosurg 55:108-116, 1981

2. Brodsky AE, Khalil MA, Sassard WR, et al: Repair of symptomatic pseudoarthrosis of anterior cervical fusion. Posterior versus anterior repair. Spine 17:1137-1143, 1992

3. Clements DH, O'Leary PF: Anterior cervical discectomy and fusion. Spine 15:1023-1025, 1990

4. Cloward RB: The anterior approach for removal of ruptured cervical disks. J Neurosurg 15:602-617, 1958

5. Eleraky MA, Llanos C, Sonntag VKH: Cervical corpectomy: report of 185 cases and review of the literature. J Neurosurg 90 (Suppl 1):35-41, 1999

6. Emery SE, Bolesta MJ, Banks MA, et al: Robinson anterior cervical fusion. Comparison of standard and modified techniques. Spine 19:660-663, 1994

7. Epstein N: The surgical management of ossification of the posterior longitudinal ligament in 51 patients. J Spinal Disord 6:432-455, 1993

8. Epstein NE: Evaluation and treatment of clinical instability associated with pseudoarthrosis after anterior cervical surgery for ossification of the posterior longitudinal ligament. Surg Neurol 49:246-252, 1998

9. Geer C, Selden NRW, Papadopoulos SM: Anterior cervical plate fixation in the treatment of single-level cervical disc disease. J Neurosurg 90:410A, 1999 (Abstract)

10. Hacker RJ: A randomized prospective study of an anterior cervical interbody fusion device with a minimum of 2 years of follow-up results. J Neurosurg 93 (Suppl 2):222-226, 2000

11. Hashizume Y, Iijima S, Kishimoto H, et al: Pathology of spinal cord lesions caused by ossification of the posterior longitudinal ligament. Acta Neuropathol 63:123-130, 1984

12. Hirabayashi K, Watanabe K, Wakano K, et al: Expansive opendoor laminoplasty for cervical spinal stenotic myelopathy. Spine 8:693-699, 1983

13. Isu T, Kamada K, Kobayashi N, et al: The surgical technique of anterior cervical fusion using bone grafts obtained from cervical vertebral bodies. J Neurosurg 80:16-19, 1994

14. Kojima T, Waga S, Kubo Y, et al: Anterior cervical vertebrectomy and interbody fusion for multi-level spondylosis and ossification of the posterior longitudinal ligament. Neurosurgery 24:864-872, 1989

15. Martin GJ, Haid RW, MacMillan M, et al: Anterior cervical discectomy with freeze-dried fibula allgraft. Overview of 317 cases and literature review. Spine 24:852-859, 1999

16. Maruo S, Kataoka O, Mizuno K: [An autopsy findings of a case of ossification of dura mater and posterior longitudinal ligament of cervical spine]. J Jap Orthop Ass 50:37-43, 1976 (Jpn)
17. Meding JB, Stambough JL: Critical analysis of strut grafts in anterior spinal fusions. J Spinal Disord 6:166-174, 1993

18. Mizuno J, Nakagawa H, Hashizume Y, et al: [Pathological study of ossification of the posterior longitudinal ligament]. (OPLL) with special reference to mechanism of ossification and spinal cord damage. Spinal Surg 2:81-87, 1988 (Jpn)

19. Mizuno J, Nakagawa H, Iwata K, et al: Pathology of the spinal cord lesions caused by ossification of the posterior longitudinal ligament, with special reference to reversibility of the spinal cord lesion. Neurol Res 14:312-314, 1992

20. Mizuno J, Nakagawa H, Isobe M: [Surgical results of anterior approach in ossification of the posterior longitudinal ligament in the cervical spine]. Spinal Surg 11:39-46, 1997 (Jpn)

21. Nagashima C: Cervical myelopathy due to ossification of the posterior longitudinal ligament. J Neurosurg 37: 653-660, 1972

22. Nakagawa H, Mizuno J: The pathophysiology and management of ossification of the posterior longitudinal ligament, in Barrow DL (ed): Perspectives in Neurological Surgery. St Louis: Quality Medical, 1992, pp 38-48

23. Nakagawa H, Mizuno J, Tamai K, et al: [Anterior approach with autogenous vertebral bone graft in ossification of the posterior longitudinal ligament of the cervical spine]. Spinal Surg 9:37-42, 1995 (Jpn)

24. Ray CD: Threaded titanium cages for lumbar interbody fusions. Spine 22:667-680, 1997

25. Savolainen S, Rinne J, Hernesniemi J: A prospective randomized study of anterior single-level cervical disc operations with long-term follow-up: surgical fusion is unnecessary. Neurosurgery 43:51-55, 1998

26. Shinomiya K, Okamoto A, Kamikozuru M, et al: An analysis of failures in primary cervical anterior spinal cord decompression and fusion. J Spinal Disord 6:277-288, 1993

27. Smith GW, Robinson RA: The treatment of certain cervicalspine disorders by anterior removal of intervertebral disc and interbody fusion. J Bone Joint Surg Am 40:607-624, 1958

28. Terayama K, Maruyama S, Miyashita R, et al: [Ossification of the posterior longitudinal ligament in the cervical spine]. Orthop Surg 15:1083-1095, 1964 (Jpn)

29. Thalgott JS, Fritts K, Giuffre JM, et al: Anterior interbody fusion of the cervical spine with coralline hydroxyapatite. Spine 24:1295-1299, 1999

30. Tsukimoto H: [A case report-autopsy of syndrome of compression of spinal cord owing to ossification within spinal canal of cervical spines]. Nippon Geka Hokan 29:1003-1007, 1960 (Jpn)

31. Williams RC: Anterior cervical fusion utilizing autogenous bone graft from the cervical vertebrae. Neurosurgeons 11: 339-342, 1992

Manuscript received February 15, 2001.

Accepted in final form March 20, 2001.

Address reprint requests to: Junichi Mizuno, M.D., Department of Neurological Surgery, Aichi Medical University 21, Karimata, Yazako, Nagakute Aichi-gun, Aichi 480-1195, Japan. email: jmizuno@amugw.aichi-med-u.ac.jp. 